\title{
A New Image Denoising Method by Combining WT with ICA
}

\author{
Chengzhi Ruan, ${ }^{1,2}$ Dean Zhao, ${ }^{1}$ Weikuan Jia, ${ }^{1}$ Chen Chen, ${ }^{1}$ Yu Chen, ${ }^{1}$ and Xiaoyang Liu ${ }^{1}$ \\ ${ }^{1}$ School of Electrical and Information Engineering, Jiangsu University, Zhenjiang 212013, China \\ ${ }^{2}$ School of Mechanical and Electrical Engineering, Wuyi University, Wuyishan 354300, China \\ Correspondence should be addressed to Dean Zhao; dazhao@ujs.edu.cn
}

Received 24 January 2015; Accepted 27 May 2015

Academic Editor: Chih-Cheng Hung

Copyright (C) 2015 Chengzhi Ruan et al. This is an open access article distributed under the Creative Commons Attribution License, which permits unrestricted use, distribution, and reproduction in any medium, provided the original work is properly cited.

\begin{abstract}
In order to improve the image denoising ability, the wavelet transform (WT) and independent component analysis (ICA) are both introduced into image denoising in this paper. Although these two algorithms have their own advantages in image denoising, they are unable to reduce noises completely, which makes it difficult to achieve ideal effect. Therefore, a new image denoising method is proposed based on the combination of WT with ICA (WT-ICA). For verifying the WT-ICA denoising method, we adopt four image denoising methods for comparison: median filtering (MF), wavelet soft thresholding (WST), ICA, and WT-ICA. From the experimental results, it is shown that WT-ICA can significantly reduce noises and get lower-noise image. Moreover, the average of WT-ICA denoising image's peak signal to noise ratio (PSNR) is improved by $20.54 \%$ compared with noisy image and $11.68 \%$ compared with the classical WST denoising image, which demonstrates its advantage. From the performance of texture and edge detection, denoising image by WT-ICA is closer to the original image. Therefore, the new method has its unique advantage in image denoising, which lays a solid foundation for the realization of further image processing task.
\end{abstract}

\section{Introduction}

Noise can be interpreted as the factor which hinders the people's sense organ from understanding and accepting the source information. In the process of the image's acquisition and transmission, due to pollution by Gaussian noise, the image quality is declined seriously. It would produce unfavorable effects on the following image processing, such as segmentation, compression, and image understanding. The purpose of image denoising is to remove noises, while keeping the main characteristics of information at the same time, such as texture and edge information of image in order to improve the quality of image. Previous studies showed that when the peak signal to noise ratio (PSNR) of a simulation image is lower than $14.2 \mathrm{~dB}$, the probability of false detection of image segmentation is more than $0.5 \%$, and the estimated error of parameters is more than $0.6 \%$ [1].

The conventional image denoising methods were based on different statistical characteristics of noise and signal, using the low-pass filters for denoising. In the spatial domain, when the statistics characteristics of noise are unknown, local smoothing operator is selected for denoising. The advantage of this method is that images can be processed in parallel, and less computation time is needed while the drawback is that the selection of window size affects the ability of denoising [2]. If the statistical characteristics of noise are known in the frequency domain, the Wiener filter [3] and least square filter [4] can be used to perform global denoising. But using this method, we need to know the statistical model of noise and signal. As we cannot use simple stochastic process to describe statistical model of image in real applications, the computational cost is relatively high. Low pass filtering method can eliminate the noise effectively, but it can also make the image edge fuzzy at the same time. There are some other denoising methods based on level set, morphological filter, and Markov model [5-7]. To reduce Gaussian noises, many scholars have proposed a series of denoising algorithm, including improved wavelet denoising method [8], improved ICA denoising method $[9,10]$, improved morphology denoising method [6, 11], method based on neural network [12], and filtering algorithm for improved denoising method [13, 14]. However, the Gaussian noise reduction was still a critical problem, as it was difficult to be removed completely. 
In this study, the wavelet transform (WT) and independent component analysis (ICA) are introduced to image denoising, and a kind of WT and ICA-based fusion method for denoising (WT-ICA) is proposed. The new method uses wavelet threshold denoising method to remove Gaussian noise in image; then, it applies ICA to separate the image maximumly into image source signal and noise; finally, it applies wavelet transform to denoise the separated image source signal again. In this way, the new denoising method can get the lower noise image as result. The WC-ICA denoising method achieves the full integration of the advantages of WT and ICA. In the experiments, to obtain different noisy images, we selected three images and added different noises. In order to better verify the denoising ability of the new method, the experiment adopts median filtering (MF), wavelet soft thresholding (WST), ICA, and WT-ICA for comparison. The experimental results indicated that the quality of processed image is greatly improved. The new method can well preserve image texture features and edge details. The PSNR is improved obviously and the obtained low noise image is more conductive for further recognition. In order to explain the effect of noise reduction, we calculate the PSNR and perform edge detection on images, the results of which demonstrate the superiority of the WT-ICA algorithm. The new method has a unique advantage in the denoising Gaussian signals in image, which lays a solid foundation for the further image processing task.

\section{Method of WT-ICA Fusion Denoising}

Gaussian white noise is considered as the main image noise. The denoising of Gaussian signal is difficult, which has attracted the attention of many scholars. They proposed numerous image denoising methods, some of which have been used to process images and reduce Gaussian noises, and some achievements have been obtained. However, these methods are still unable to reach the ideal denoising effect [15, 16]. In this study, the WT and ICA are introduced into image processing simultaneously. In order to reduce noise pollution of images to the maximum extent, this study tries to combine these two methods together for image denoising.

2.1. Wavelet Denoising. Wavelet transform has good properties of localization both in the time and frequency domains. This characteristic can not only characterize the texture and structure of images at different resolution levels, but also contributes to the edge detection. Therefore, wavelet-based denoising can extract and preserve the edge information, which plays an important role in vision at the same time. It takes the lead in realizing nonlinear transform denoising of image. It is one of the hotspots in image processing field, and more and more new methods are proposed.

All the wavelet denoising methods follow the basic principle: the wavelet coefficients of image source signal and noise have different properties at different scales. By constructing the corresponding evaluation criteria, WT uses mathematical methods to process the corresponding wavelet coefficients of noise signal in the wavelet domain. This study adopts the wavelet threshold denoising method, and the evaluation

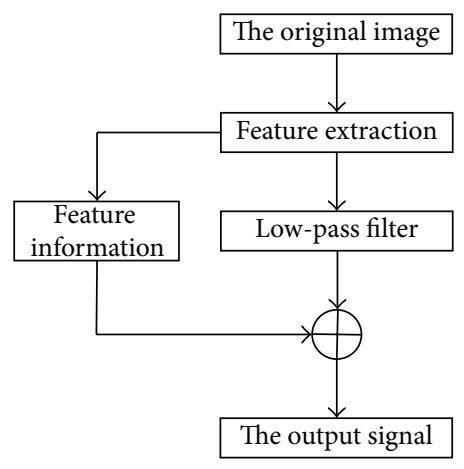

FIGURE 1: Flow chart of wavelet denoising.

criterion is the predetermined threshold. That is, we process wavelet coefficients according to a predetermined threshold. If wavelet coefficients are less than a predetermined threshold, these coefficients resulting from the noise thus can be ignored. Otherwise, it is regarded that wavelet coefficients are caused by the image signal source; thus, these coefficients are kept or expanded, and then we reconstruct and restore them. Finally, the low noise image is obtained.

On the one hand, from the mathematical point of view, wavelet denoising belongs to function approximation in the essence. On the other hand, it can seem as a signal filtering problem when it is analyzed from the signal perspective. Therefore, the wavelet denoising is actually an integration of image features extraction and image low-pass filter. Figure 1 is the flow chart of wavelet denoising.

Suppose the noisy image signal is

$$
f(i, j)=s(i, j)+n(i, j),
$$

where $s(i, j)$ is the image source signal and $n(i, j)$ is the noise signal that follows $N\left(0, \sigma^{2}\right)$. When performing the discrete wavelet transform of observational image signal $f(i, j)$, the wavelet coefficients are also composed of two parts: the corresponding wavelet coefficients of image source signal and the corresponding wavelet coefficients of noise signal.

The essence of image threshold denoising is to apply onedimensional wavelet transform to rows and columns of image and then to perform threshold denoising of one-dimensional signal. When making discrete sampling to the row vector as in formula (1), $N$ discrete points signal $f(n)$ can be obtained. The discrete wavelet transform is

$$
W f(\alpha, k)=2^{-\alpha / 2} \sum_{n=0}^{N-1} f(n) \psi\left(2^{-\alpha} n-k\right) .
$$

In the above formula, $W f(\alpha, k)$ is the wavelet coefficient, and $\alpha$ is decomposition scale. Similarly, the wavelet transform is performed on the column vectors.

This study adopts wavelet soft threshold denoising method, the basic idea of which is carried out as follows.

Step 1. Apply wavelet transform to the two-dimensional image signal, select the appropriate wavelet basis and decomposition layer $\alpha$, and calculate the decomposition of signal $f$ to the $\alpha$ layer. 


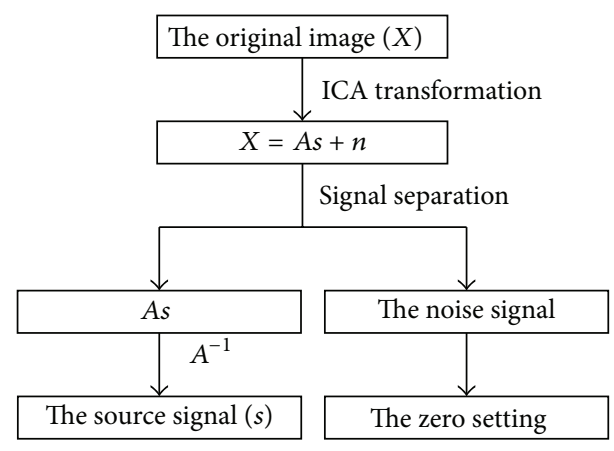

FIgURE 2: Flow chart of ICA denoising.

Step 2. Conduct the threshold quantization to the decomposed high frequency coefficients, choose an appropriate threshold for each layer $1 \sim \alpha$, and make the soft threshold quantization to the high frequency coefficients of the layer.

Step 3. Calculate the reconstruction of two-dimensional signal according to the low frequency coefficients in the $\alpha$ layer of the wavelet decomposition and the high frequency coefficient modified from 1 to $\alpha$ layer of each layer, and obtain the low noise image.

When the absolute value of the wavelet coefficients is larger than a given threshold, let the soft threshold function minus the threshold; otherwise, it is set to 0 . This is described as

$$
\widetilde{w}_{\alpha, k}= \begin{cases}\operatorname{sgn}\left(w_{\alpha, k}\right) \cdot\left(\left|w_{\alpha, k}\right|-\lambda\right), & \left|w_{\alpha, k}\right|>\lambda, \\ 0, & \left|w_{\alpha, k}\right| \leq \lambda .\end{cases}
$$

Among them, in the threshold function, $w_{\alpha, k}$ is the $k$ th wavelet coefficients in the $\alpha$ scale, $\widetilde{w}_{\alpha, k}$ is the wavelet coefficient after treatment with threshold function, and $\lambda$ is the threshold defined as

$$
\lambda=\sigma \sqrt{2 \ln M \times N},
$$

where $\sigma$ is noise standard deviation. $M$ and $N$ are rows and columns of the image pixel, respectively.

2.2. ICA Denoising. ICA is widely used in the blind source signal separation, such as the cocktail party problem $[17,18]$. As the development of ICA research, it has broad applications $[19,20]$. Under certain conditions, ICA can well separate hidden source signal from mixed signal. When it is used for image processing, the main task is to extract image source signal from noisy image and to achieve the denoising effect. The basic idea of ICA noise reduction is to regard the noisy image as a mixture of two independent signal sources which are image source signal and noise signal. For image denoising, ICA separates the image source signal from the noise signal with less loss of image details and achieves the purpose of noise reduction.

The flow chart of ICA denoising is shown in Figure 2.

Assume that the ICA model with noise is

$$
x=A s+n .
$$

Among them, $x$ is the observed image signal, $s=\left[s_{1}, s_{2}\right.$, $\left.\ldots, s_{n}\right]^{T}$ is the image source signal, $n=\left[n_{1}, n_{2}, \ldots, n_{m}\right]^{T}$ is the noise signal, and $A$ is a mixed matrix of order $m \times n$. $s$ and $n$ are independent of each other.

The mixing matrix is estimated by the method of maximum likelihood. However, in the ICA model with noise, it is not enough to estimate only the mixing matrix. By the inversion of formula (5), we can get

$$
W x=s+W n .
$$

This is the estimation of independent component which contains noise. However, it is expected that the estimation is an independent component of source signal $\widehat{s}_{i}$, and the component is allowed to achieve optimum in some extent, that is, to contain the minimum noise.

This study uses FICA algorithm for image denoising. The basic steps are as follows.

Step 1. Use the training set $v$ without noise to estimate the ICA base vector of $v$, where $v=A s$. Obtain estimations of union $\widehat{A}$ and order $W_{0}=\widehat{A}^{-1}$; then, calculate $W=$ $W_{0}\left(W_{0}^{T} W_{0}\right)^{-1 / 2}$.

Step 2. Estimate each component probability density $s_{i}=$ $w_{i}^{T} v$. Apply ICA transform to the original signal with noise, and calculate the sparse transform projection $Y=W \widehat{X}$ based on transform matrix $W$.

Step 3. Use the maximum likelihood function of contraction $g_{i}$ to get denoising estimation $s_{i}=g_{i}\left(y_{i}\right)$.

Step 4. Take an inversion to transform; then, get low image noise estimation $\widehat{X}=W^{T} \widehat{S}$.

Thus, it can be seen that ICA is to decompose the image under certain criterion into a series of basis vectors that are not related to each other, and then choose a certain amount of basis vectors to reconstruct image referring to the criterion which finally achieves the image denoising.

\subsection{WT-ICA Denoising}

2.3.1. The Idea of Fusion Denoising. In order to achieve better denoising performance, this study further combines wavelet transform with ICA and proposes a new denoising method based on WT and ICA (WT-ICA). Given the original signal $X$ of image with noise, the new method first uses wavelet soft threshold denoising algorithm to remove the part of Gaussian noise, which can be regarded as the first image denoising. In order to minimize information loss, it cannot perform continuous wavelet transform denoising. The ICA is applied to the obtained images with less noise to make a maximum separation of the image source signal and noise signal; then, it completes the ICA denoising. The image processed by ICA can maintain a low distortion. However, there still exist some noises in the current images obtained; thus, the wavelet soft threshold denoising is used again to obtain higher-quality image source signal. 


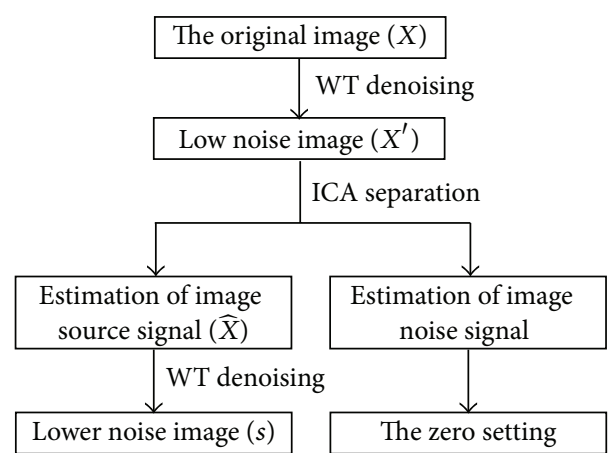

FIGURE 3: Flow chart of WT-ICA denoising.

We apply the wavelet soft threshold denoising again, which is different from the classical second wavelet denoising. For the two wavelet threshold denoising methods in this study, we make independent component analysis on the low noise image which is obtained from the first step in order to maximize the elimination of noise interference and enhance the denoising performance.

2.3.2. The Basic Steps of the Fusion Algorithm. In this study, based on the idea of WT and ICA integration, we propose the WT-ICA fusion method for image denoising. The flow chart is shown in Figure 3.

The basic steps of fusion image denoising are as follows.

Step 1. Apply wavelet transform to the two-dimensional image signal, and calculate the decomposition of signal $X$ in the $\alpha$ layer.

Step 2. Choose an appropriate threshold for each layer $1 \sim \alpha$, and conduct the soft threshold quantization to the high frequency coefficients of the layer.

Step 3. Calculate the reconstruction of two-dimensional signal according to the low frequency coefficients in the $\alpha$ layer of the wavelet decomposition and the high frequency coefficient modified from the first to the $\alpha$ layer of each layer to get low noise image $X^{\prime}$.

Step 4. Use source image training set without noise to estimate ICA-based vector and get the mixing matrix $A$.

Step 5. Apply ICA transform and the maximum likelihood estimation to remove the noise estimation for low noise image.

Step 6. Apply inverse transform of ICA to get image source signal estimation $\widehat{X}$.

Step 7. Repeat Steps 1-3 to get lower noise image.

\section{Experimental of Image Denoising}

3.1. The Original Image. In order to validate the new method, we selected three groups of images in the experiments, as shown in Figure 4. The pixel of standard lenna image in Figure 4 (a) is $512 \times 512$. To further verify the performance, the Red Fuji apple image is selected, as shown in Figure 4(b) which is captured by a certain type of apple harvesting robot in natural environment. The camera model is the SONY CYBERSHOT. The scenic image in Figure 4(c) is captured by CANON ESO 600 D. For subsequent validation of image denoising, three representative images are selected. These three color images are converted to grayscale images, which are shown in Figure 5.

3.2. Noise Reduction Experiments. The experimental operation platform in this study is described as follows: the host configuration: CPU Intel Core2 Duo E7300 2.66 GHz, RAM $1.99 \mathrm{~GB}$, Intel graphics G33/G31 ECF runtime environment: MATLAB R2012a.

In order to better verify the new method, the following denoising methods are adopted in the experiment: median filtering denoising method (MF), wavelet soft denoising method (WST), ICA denoising method, and WT-ICA denoising method. These four denoising methods were compared to each other. The window size for MF method is $3 * 3$. The decomposition scale for WSF denoising method is 3 . The window size for orthogonal ICA transform is $8 * 8$.

3.2.1. Experiment 1. In order to better verify the validation of the proposed denoising method, the denoising comparison among four methods on the standard image of lenna is illustrated in Figure 6. The lenna gray image is superimposed to Gaussian white noise with zero mean and different intensities $\left(\sigma_{n}{ }^{2}=0.001,0.01,0.05,0.1\right)$ so that the noisy images are obtained. Under different noise levels, as shown in Figure 6, we get noise reduction results by using different denoising methods. Figures 6(a)-6(d) list the images with noise and noise reduction results with the condition of ${\sigma_{n}}^{2}$ equal to $0.001,0.01,0.05$, and 0.1 . From the first to the fifth columns of each row, they are noisy image (NI), denoising results obtained by median filtering (MF) method, WST denoising method, ICA denoising method, and WT-ICA denoising method, respectively.

3.2.2. Experiment 2. In order to further verify the effectiveness of our algorithm, the Gaussian white noises with zero mean and different intensities $\left(\sigma_{n}{ }^{2}=0.01,0.05,0.1\right)$ are superimposed on the gray image of apple, respectively, to get noisy images. Then, a comparison is made among noisy images obtained by using median filtering denoising, soft threshold denoising, ICA denoising, and WT-ICA noise reduction method, respectively. The above four denoising methods are used to denoise the added noisy image. The denoising results are shown in Figures $7(a)-7(\mathrm{c})$. The order of Figure 7 is the same as that in Figure 6.

3.2.3. Experiment 3. We conduct the experiment 3 with the mountain image. The procedure of experiment 3 is the same as that of the experiment 2 . The denoising results are shown in Figures 8(a)-8(c). 


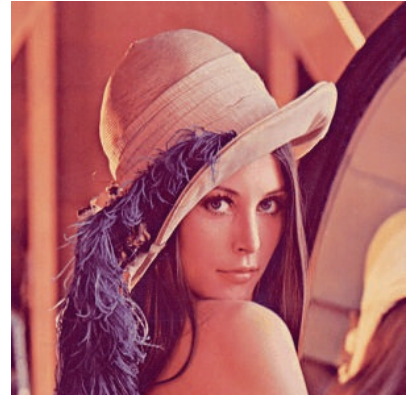

(a) Lenna

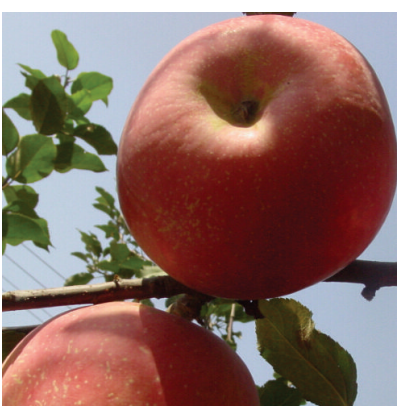

(b) Apple

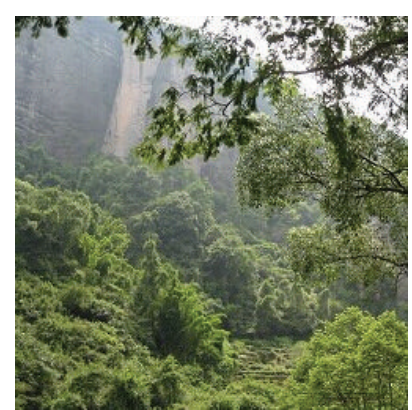

(c) Mountain

FIGURE 4: The original color images.

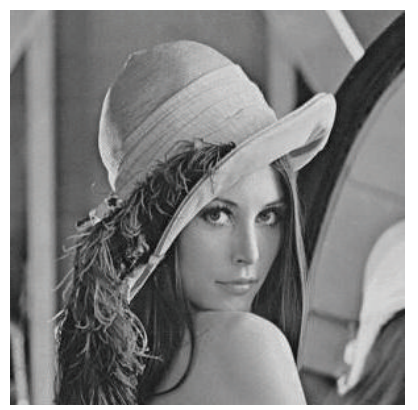

(a) Lenna

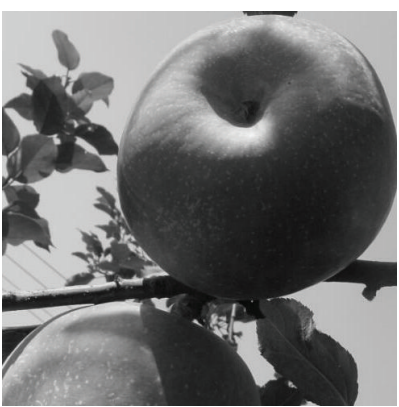

(b) Apple

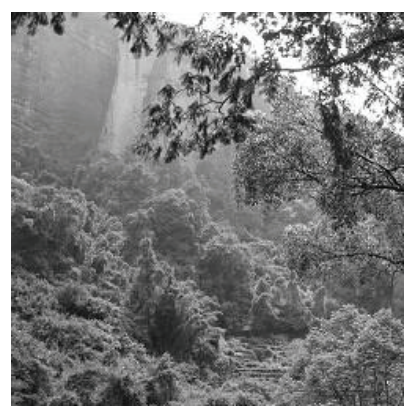

(c) Mountain

FIGURE 5: The original gray images.

\subsection{Experimental Results and Analysis}

3.3.1. Visual Analysis. In Figures 6-8, from the visual point of view, it can be observed that four kinds of denoising methods can reduce noises to a certain extent. But by comparing the low noise images obtained from four kinds of denoising methods, it can be seen that the denoising performance of MF method is relatively worse, the results of which retain more noises and are fuzzy. The denoising results of the WST denoising method and ICA denoising method are clearer than the results of the MF denoising method, but there are still some noises existing and the denoising is incomplete. Therefore, to some extent, it needs to be further improved. Compared to other three methods for noise reduction, the images obtained by WT-ICA denoising method are the clearest with lowest noise, the performance of which is the best.

3.3.2. The PSNR Evaluation. WT-ICA denoising method greatly improves the performance of image restoration. The visual quality has been greatly improved as well. However, as the evaluation by human vision is subjective, it cannot verify the real denoising performance in a fair and objective way.

In order to measure the performance of denoising algorithm objectively, the PSNR of different images, mean square error (MSE) of lenna, apple and mountain images by using four denoising methods are listed in Tables 1 and 2, respectively. These results are the average results of 20 times experiments.

From both the results of PSNR and MSE, it can be observed that the method proposed in this paper has obvious
TABLE 1: The PSNR comparison of different noise reduction methods.

\begin{tabular}{ccccccc}
\hline Image & $\sigma_{n}{ }^{2}$ & $\mathrm{NI}$ & $\mathrm{MF}$ & $\mathrm{WST}$ & $\mathrm{ICA}$ & WT-ICA \\
\hline \multirow{4}{*}{ Lenna } & 0.001 & 30.2486 & 32.8937 & 31.2977 & 31.3042 & 35.8509 \\
& 0.01 & 29.6274 & 28.4660 & 30.7974 & 29.7149 & 33.3217 \\
& 0.05 & 27.7434 & 28.6445 & 30.1754 & 27.8348 & 33.1302 \\
& 0.1 & 27.5489 & 28.2028 & 29.0964 & 28.2738 & 32.2946 \\
\hline \multirow{4}{*}{ Apple } & 0.01 & 28.6552 & 31.0486 & 31.7073 & 31.8736 & 35.5676 \\
& 0.05 & 27.7477 & 31.0147 & 29.8020 & 31.8391 & 35.0163 \\
& 0.1 & 27.5549 & 28.2936 & 30.1613 & 28.8020 & 33.1680 \\
\hline \multirow{4}{*}{ Mountain } & 0.01 & 27.8586 & 30.2643 & 30.5784 & 31.0982 & 33.6078 \\
& 0.05 & 26.6541 & 30.0129 & 29.8475 & 28.8976 & 32.7673 \\
& 0.1 & 25.9783 & 27.8951 & 28.1496 & 28.9571 & 32.0735 \\
\hline \multirow{6}{*}{} & & & & & &
\end{tabular}

advantages compared with other methods. The PSNR is improved by about $5 \sim 8 \mathrm{~dB}$, especially in high noise intensity; that is, the signal-to-noise ratio is low, where the effect is more obvious and the advantage of this method can be reflected more directly. The average of WT-ICA denoising image's PSNR is improved by $20.54 \%$ compared with noisy image, and $11.68 \%$ compared with the classical WST denoising image. Therefore, the advantage can be demonstrated obviously.

3.3.3. Texture Features Comparison. In order to verify the characteristics of retaining image texture in this paper, the joint statistical distribution of pixel and the edge gradient 

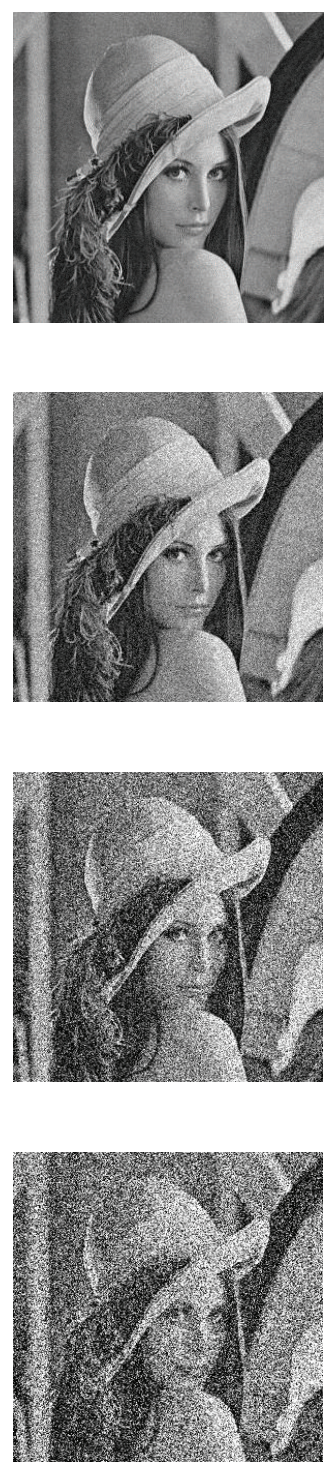
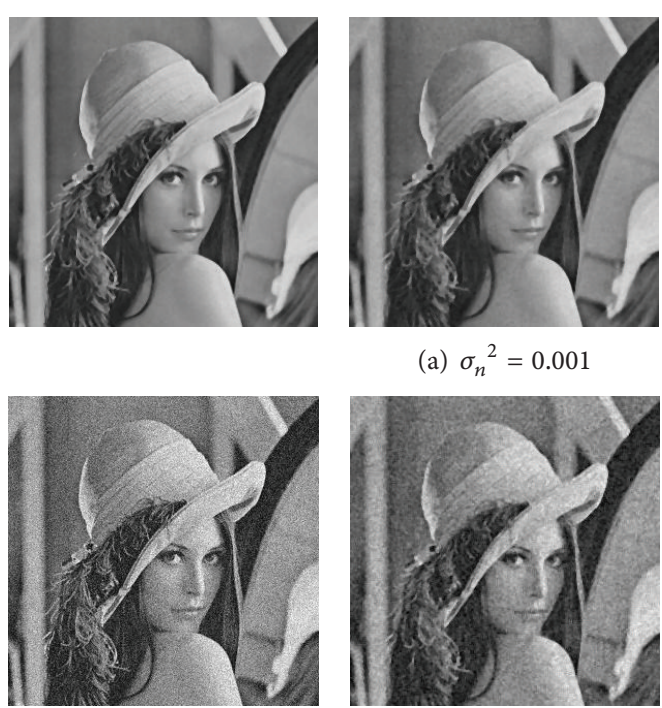

(a) $\sigma_{n}^{2}=0.001$

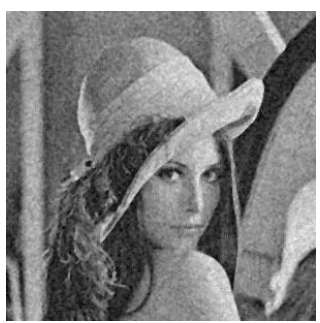

(b) $\sigma_{n}^{2}=0.01$

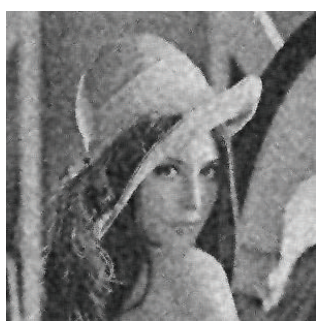

(c) $\sigma_{n}^{2}=0.05$
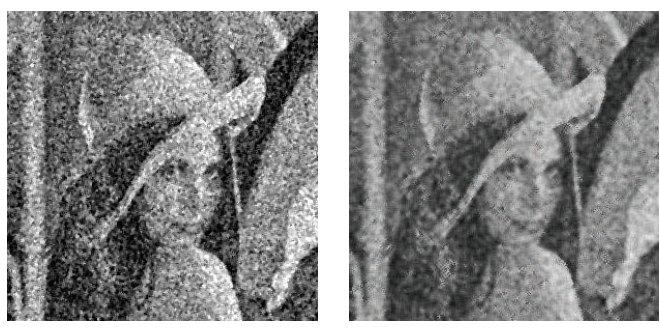
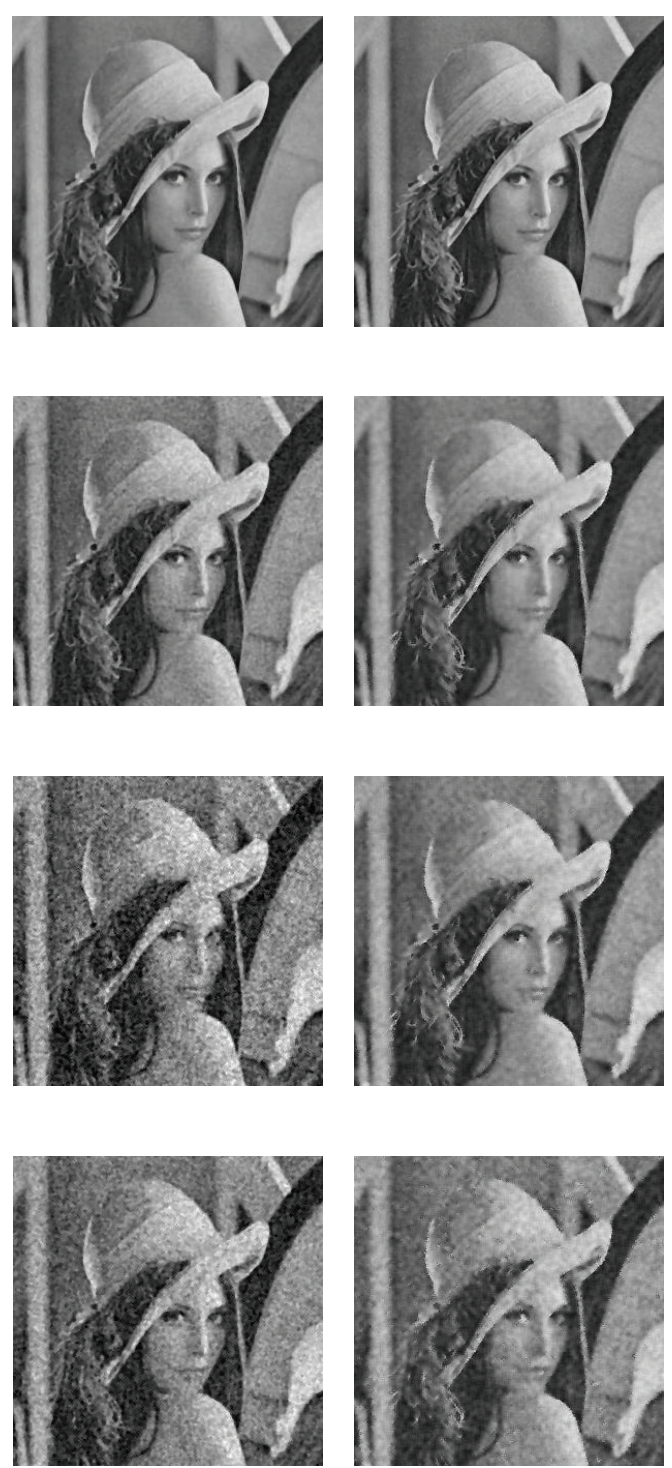

(d) $\sigma_{n}^{2}=0.1$

FIgure 6: The denoising results of lenna.

TABLE 2: The MSE comparison of different noise reduction methods.

\begin{tabular}{ccccccc}
\hline Image & $\sigma_{n}{ }^{2}$ & NI & MF & WST & ICA & WT-ICA \\
\hline \multirow{4}{*}{ Lenna } & 0.001 & 61.4078 & 33.3969 & 48.2288 & 48.1575 & 16.9040 \\
& 0.01 & 70.8502 & 92.5712 & 54.1181 & 69.4370 & 30.2629 \\
& 0.05 & 109.3291 & 88.8448 & 62.4513 & 107.0526 & 31.6272 \\
& 0.1 & 114.3387 & 98.3551 & 80.0639 & 96.7604 & 38.3372 \\
\hline \multirow{4}{*}{ Apple } & 0.01 & 88.6262 & 51.0761 & 43.8886 & 42.2396 & 18.0435 \\
& 0.05 & 109.2227 & 51.4765 & 68.0582 & 42.5766 & 20.4857 \\
& 0.1 & 114.1801 & 96.3210 & 62.6538 & 85.6795 & 31.3531 \\
\hline \multirow{4}{*}{ Mountain } & 0.01 & 106.4683 & 61.1858 & 56.9168 & 50.4964 & 28.3335 \\
& 0.05 & 140.4981 & 64.8321 & 67.3489 & 83.8147 & 34.3835 \\
& 0.1 & 164.1536 & 105.5773 & 99.5681 & 82.6743 & 40.3395 \\
\hline
\end{tabular}

with four kinds of denoising methods are calculated, and the gray gradient cooccurrence matrix is used to extract image texture features [21]. Taking lenna image with noised intensity $\sigma_{n}{ }^{2}=0.01$ as example, we calculate four typical texture features: energy $T_{1}$, correlation $T_{2}$, grey entropy $T_{3}$, and moment of inertia $T_{4}$, as shown in Table 3. In Table 3, OI (original image) is the representation of the original image, and the other codes are the same as Table 1. From the results, the texture features of image processed by WT-ICA denoising method is closest to the original image, so the WT-ICA denoising method is the best in retaining texture features.

3.3.4. Comparison on Edge Detection. In order to further verify the feasibility of four methods of image denoising, noise intensity $\sigma_{n}^{2}=0.01$ is added to the lenna image (original image, noised image, and image denoising of four kinds of methods). The results of edge detection are shown in Figure 9. The Canny edge detection operator is the most commonly used edge detection operator, which is recognized as 
TABLE 3: The typical texture features comparison among different denoising methods.

\begin{tabular}{|c|c|c|c|c|c|c|}
\hline Typical texture features & OI & $\mathrm{NI}$ & MF & WST & ICA & WT-ICA \\
\hline$\overline{T_{1}}$ & 0.0011 & 0.0021 & 0.0018 & 0.0008 & 0.0008 & 0.0009 \\
\hline$T_{2}$ & -135.6152 & -286.1320 & -203.8348 & -97.6614 & -83.7051 & -125.7223 \\
\hline$T_{3}$ & 2.2555 & 2.3420 & 2.3105 & 2.2743 & 2.2647 & 2.2459 \\
\hline$T_{4}\left(\times 10^{-4}\right)$ & 1.5099 & 1.4299 & 1.4373 & 1.4195 & 1.3884 & 1.4897 \\
\hline
\end{tabular}
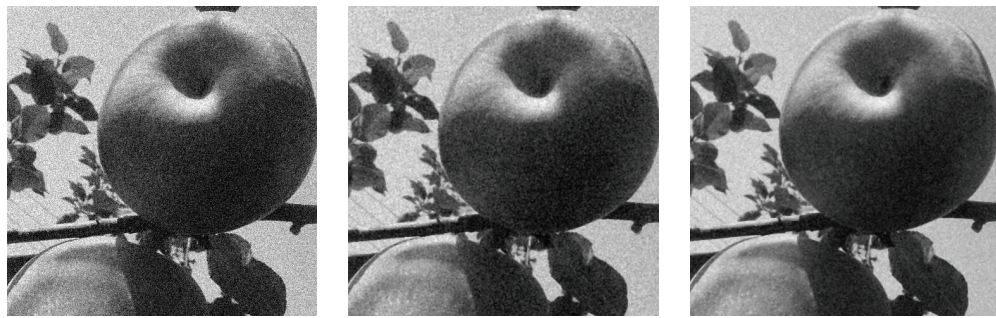

(a) $\sigma_{n}{ }^{2}=0.01$
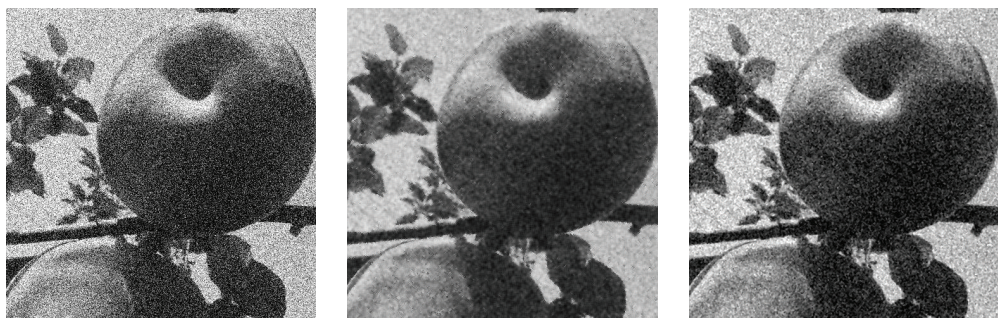

(b) $\sigma_{n}^{2}=0.05$
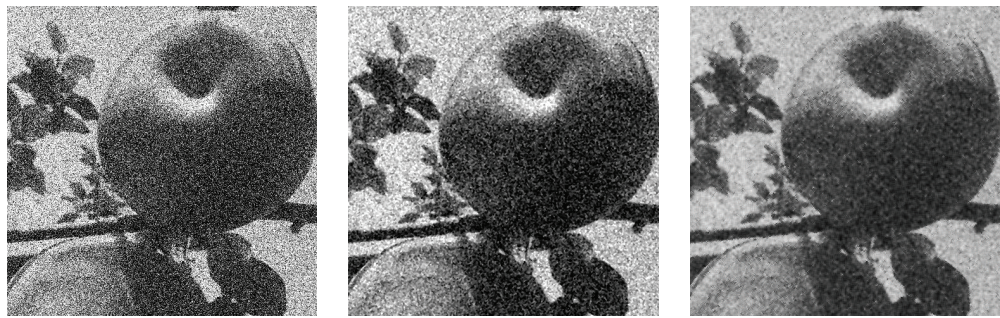
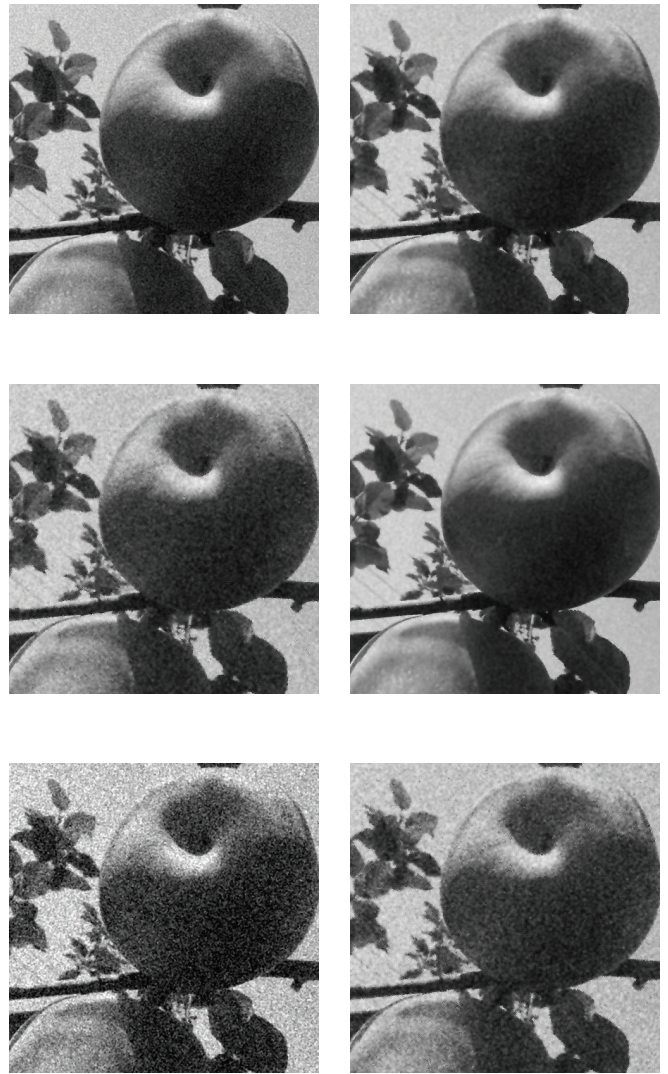

(c) $\sigma_{n}^{2}=0.1$

Figure 7: The denoising results of apple.

an excellent detection operator [22]. From Figure 9, it can be seen that the edge detection results of WT-ICA denoising method is able to extract the whole contour information of the image, which is closer to the original image. Thus, the proposed method is more effective to keep image texture and edge details.

Through these experiments, we compare and analyze noise reduction ability and the denoising effect of four different methods in Figures 5-9 and Tables 1-3, where the performances are evaluated from the visual and objective aspects, respectively. All the results in this study show that the effect of WT-ICA denoising method proposed is the optimal.

\section{Conclusion and Discussion}

The image denoising for Gaussian noise is a difficult problem in the image processing. For this task, the wavelet transform and independent component analysis are introduced into image denoising at the same time. These two algorithms have their own advantages in image denoising. However, it is difficult to achieve the ideal effect as noise reduction is incomplete. In order to better reduce the noise, we combine the two advantages of WT and ICA, and a new image denoising method based on WT and ICA (WT-ICA) is proposed. The experimental results show that the noises of low noise image are significantly reduced by using WST and ICA noise reduction, where the resulting image is clearer and the PSNR is improved. The denoising effects of these two methods are slightly better than those of the median filter denoising method. However, these two methods of image denoising are still incomplete, which need to be further improved. The quality of image processed by WT-ICA image denoising method is greatly improved, where the value of MSE is further reduced, and the PSNR about $1-5 \mathrm{~dB}$ is improved. This new method retains image texture and edge details very well, and the obtained low noise image is more conducive for 

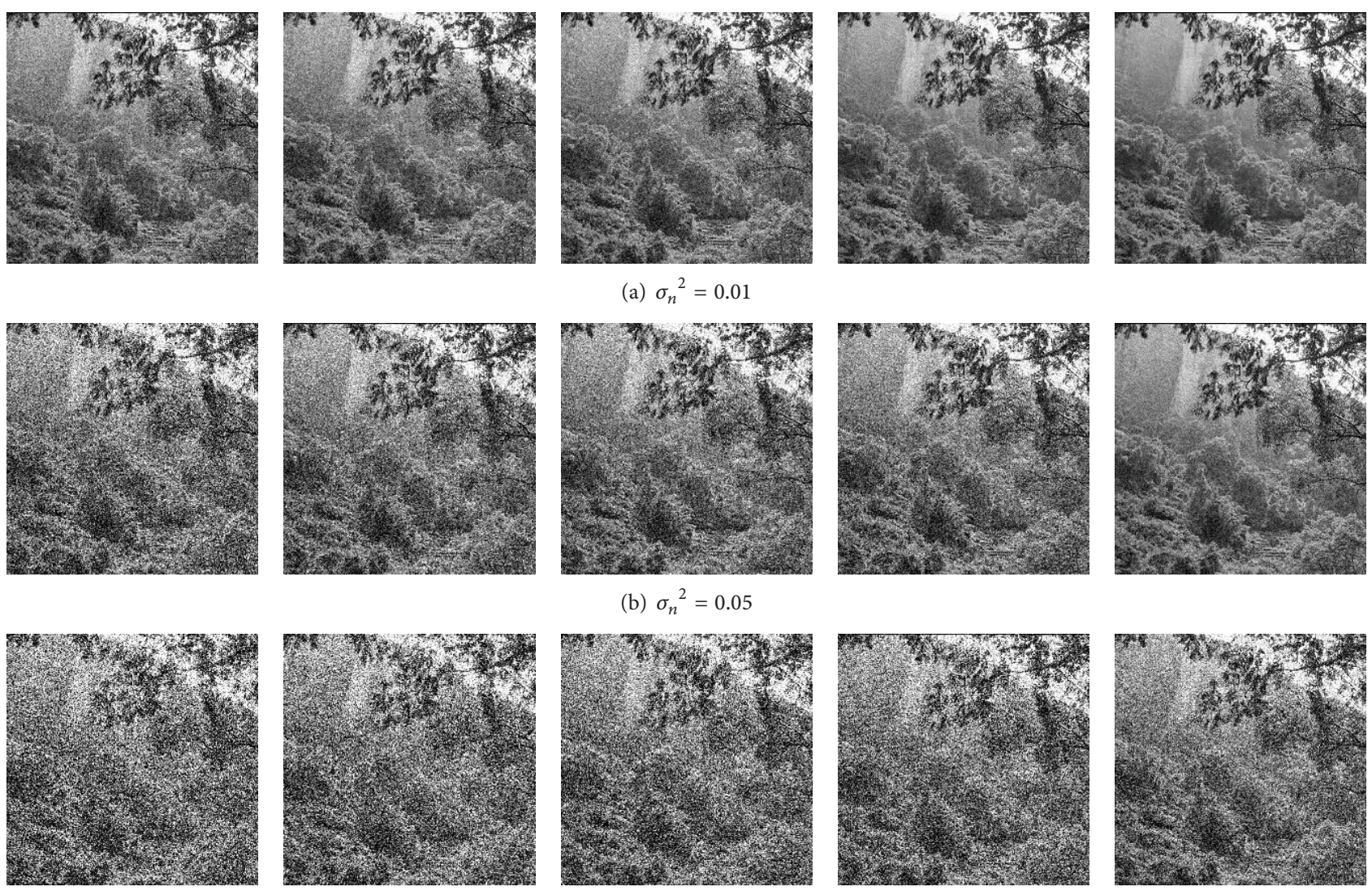

(c) $\sigma_{n}^{2}=0.1$

FIGURE 8: The denoising results of mountain.

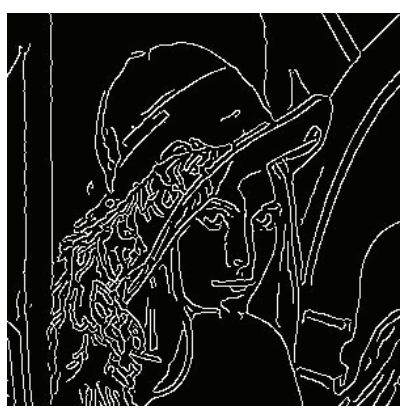

(a) $\mathrm{OI}$

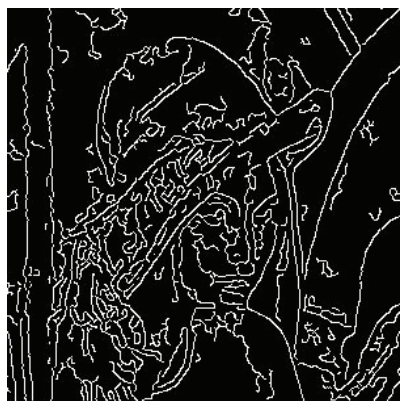

(d) WST

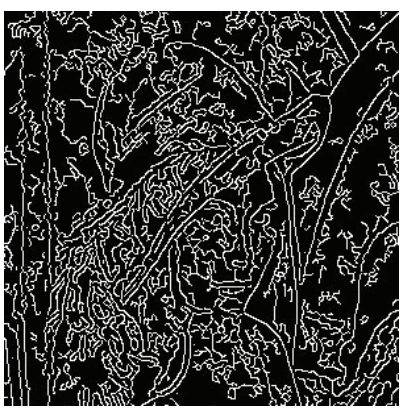

(b) NI

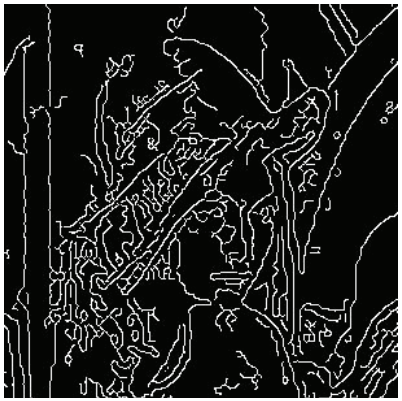

(e) ICA

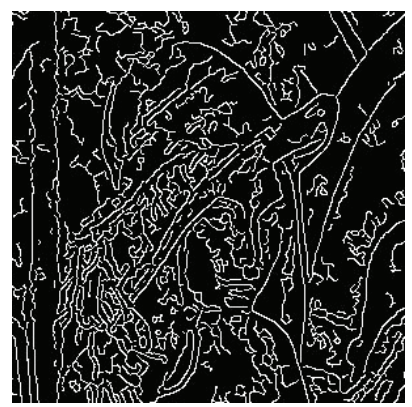

(c) MF

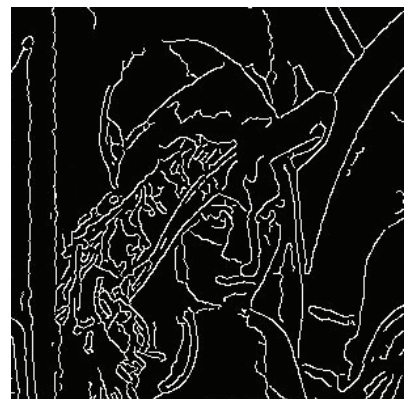

(f) WT-ICA

Figure 9: Canny edge detector (lenna ${\sigma_{n}}^{2}=0.01$ ). Note: the symbols in Figure 9 are the same as Table 1. 
the further recognition and processing. It is demonstrated that WT-ICA denoising method is feasible. The effect is more obvious especially in the case of high noise intensity, that is, the signal-to-noise ratio is low.

Overall, there are lots of problems for image denoising yet to be explored and solved. These problems should be dealt with according to the specific image noise type by using modern electronic equipment, such as spectrum analyzer, or by selecting appropriate algorithms as image denoising method $[23,24]$ such as partial differential equations that can determine types of complicated noise. WT-ICA has become one kind of research methods for image denoising. In this paper, only Gaussian noise is discussed and the research on non-Gaussian noise is waiting for further verification. Many experts have turned to this research area, so it is necessary to generalize the research results of Gauss noise to that of nonGaussian noise, which is still a difficult problem at present.

In machine vision, image denoising is an important step in image processing. There are many other problems yet to be solved, such as segmentation, recognition, and visual navigation problems, which restrict the development of image processing technology and need to be further studied.

\section{Conflict of Interests}

The authors declare that there is no conflict of interests regarding the publication of this paper.

\section{Acknowledgments}

This work is supported by the National Natural Science Foundation of China (nos. 61203014 and 61379101), Priority Academic Program Development of Jiangsu Higher Education Institutions, Major Projects in the National Science \& Technology Pillar Program during the Twelfth Five-Year Plan Period (no. 2011BAD20B06), The Specialized Research Fund for the Doctoral Program of Higher Education of China (no. 20133227110024), and the Ordinary University Graduate Student Research Innovation Projects of Jiangsu Province (no. KYLX14_1062).

\section{References}

[1] T. Lei and J. K. Udupa, "Performance evaluation of finite normal mixture model-based image segmentation techniques," IEEE Transactions on Image Processing, vol. 12, no. 10, pp. 1153-1169, 2003.

[2] H. Talebi, X. Zhu, and P. Milanfar, "How to saif-ly boost denoising performance," IEEE Transactions on Image Processing, vol. 22, no. 4, pp. 1470-1485, 2013.

[3] R. Y. Xia and C. C. Bao, "Wiener filtering based speech enhancement with weighted denoising auto-encoder and noise classification," Speech Communication, vol. 60, pp. 13-29, 2014.

[4] Y. Li, J. Li, L. Wang, J. Zhang, D. Li, and M. Zhang, "A weighted least squares algorithm for time-of-flight depth image denoising," Optik, vol. 125, no. 13, pp. 3283-3286, 2014.

[5] Y. Kuang, L. Zhang, and Z. Yi, "An adaptive rank-sparsity KSVD algorithm for image sequence denoising," Pattern Recognition Letters, vol. 45, no. 1, pp. 46-54, 2014.
[6] M. Sharif, M. Arfan Jaffar, and M. Tariq Mahmood, "Optimal composite morphological supervised filter for image denoising using genetic programming: application to magnetic resonance images," Engineering Applications of Artificial Intelligence, vol. 31, pp. 78-89, 2014.

[7] J. Villa, R. Rodríguez-Vera, J. A. Quiroga, I. de la Rosa, and E. González, "Anisotropic phase-map denoising using a regularized cost-function with complex-valued Markov-randomfields," Optics and Lasers in Engineering, vol. 48, no. 6, pp. 650656, 2010.

[8] M. Boix and B. Cantó, "Using wavelet denoising and mathematical morphology in the segmentation technique applied to blood cells images," Mathematical Biosciences and Engineering, vol. 10, no. 2, pp. 279-294, 2013.

[9] V. Nassiri, M. Aminghafari, and A. Mohammad-Djafari, "Solving noisy ICA using multivariate wavelet denoising with an application to noisy latent variables regression," Communications in Statistics: Theory and Methods, vol. 43, no. 10-12, pp. 2297-2310, 2014.

[10] T. Saba, A. Rehman, A. Al-Dhelaan, and M. Al-Rodhaan, "Evaluation of current documents image denoising techniques: a comparative study," Applied Artificial Intelligence, vol. 28, no. 9, pp. 879-887, 2014.

[11] M. B. Ashtiani and S. M. Shahrtash, "Feature-oriented denoising of partial discharge signals employing mathematical morphology filters," IEEE Transactions on Dielectrics and Electrical Insulation, vol. 19, no. 6, pp. 2128-2136, 2012.

[12] S. Bhattacharyya, P. Pal, and S. Bhowmick, "Binary image denoising using a quantum multilayer self organizing neural network," Applied Soft Computing, vol. 24, pp. 717-729, 2014.

[13] B. K. K. Shreyamsha, "Image denoising based on gaussian/ bilateral filter and its method noise thresholding," Signal, Image and Video Processing, vol. 7, no. 6, pp. 1159-1172, 2013.

[14] R. M. Yan, L. Shao, L. Liu, and Y. Liu, "Natural image denoising using evolved local adaptive filters," Signal Processing, vol. 103, pp. 36-44, 2014.

[15] N. Remenyi, O. Nicolis, G. Nason, and B. Vidakovic, "Image denoising with 2D scale-mixing complex wavelet transforms," IEEE Transactions on Image Processing, vol. 23, no. 12, pp. 51655174, 2014.

[16] D. P. Jena and R. Kumar, "Implementation of wavelet denoising and image morphology on welding image for estimating HAZ and welding defects," Measurement Science Review, vol. 11, no. 4, pp. 108-111, 2011.

[17] H. Sawada, S. Araki, R. Mukai, and S. Makino, "Blind extraction of dominant target sources using ICA and time-frequency masking," IEEE Transactions on Audio, Speech and Language Processing, vol. 14, no. 6, pp. 2165-2173, 2006.

[18] T. Kim, H. T. Attias, S.-Y. Lee, and T.-W. Lee, "Blind source separation exploiting higher-order frequency dependencies," IEEE Transactions on Audio, Speech and Language Processing, vol. 15, no. 1, pp. 70-79, 2007.

[19] G. Salimi-Khorshidi, G. Douaud, C. F. Beckmann, M. F. Glasser, L. Griffanti, and S. M. Smith, "Automatic denoising of functional MRI data: combining independent component analysis and hierarchical fusion of classifiers," NeuroImage, vol. 90, pp. 449-468, 2014.

[20] J. Coloigner, L. Albera, A. Kachenoura, F. Noury, and L. Senhadji, "Semi-nonnegative joint diagonalization by congruence and semi-nonnegative ICA," Signal Processing, vol. 105, pp. 185197, 2014. 
[21] Y.-Y. Liao, J.-C. Wu, C.-H. Li, and C.-K. Yeh, "Texture feature analysis for breast ultrasound image enhancement," Ultrasonic Imaging, vol. 33, no. 4, pp. 264-278, 2011.

[22] Q. Xu, S. Varadarajan, C. Chakrabarti, and L. J. Karam, "A distributed canny edge detector: algorithm and FPGA implementation," IEEE Transactions on Image Processing, vol. 23, no. 7, pp. 2944-2960, 2014.

[23] A. A. Yahya, J. Q. Tan, and M. Hu, "A blending method based on partial differential equations for image denoising," Multimedia Tools and Applications, vol. 73, no. 3, pp. 1843-1862, 2014.

[24] E. Nadernejad and M. Nikpour, "Image denoising using new pixon representation based on fuzzy filtering and partial differential equations," Digital Signal Processing, vol. 22, no. 6, pp. 913-922, 2012. 


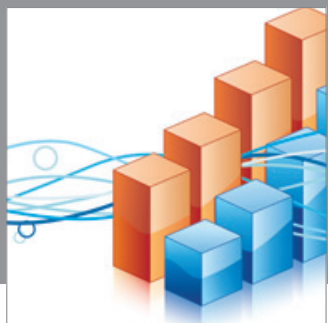

Advances in

Operations Research

mansans

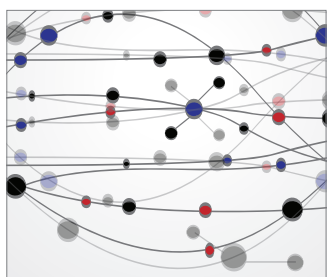

The Scientific World Journal
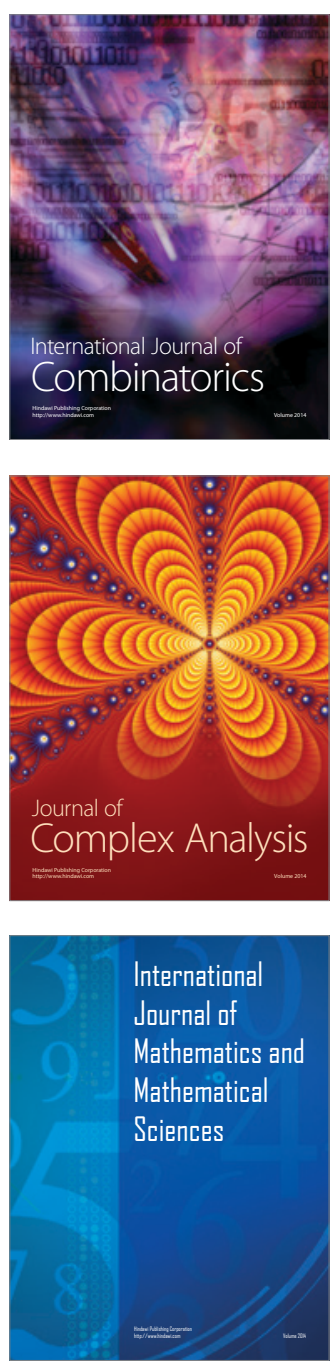
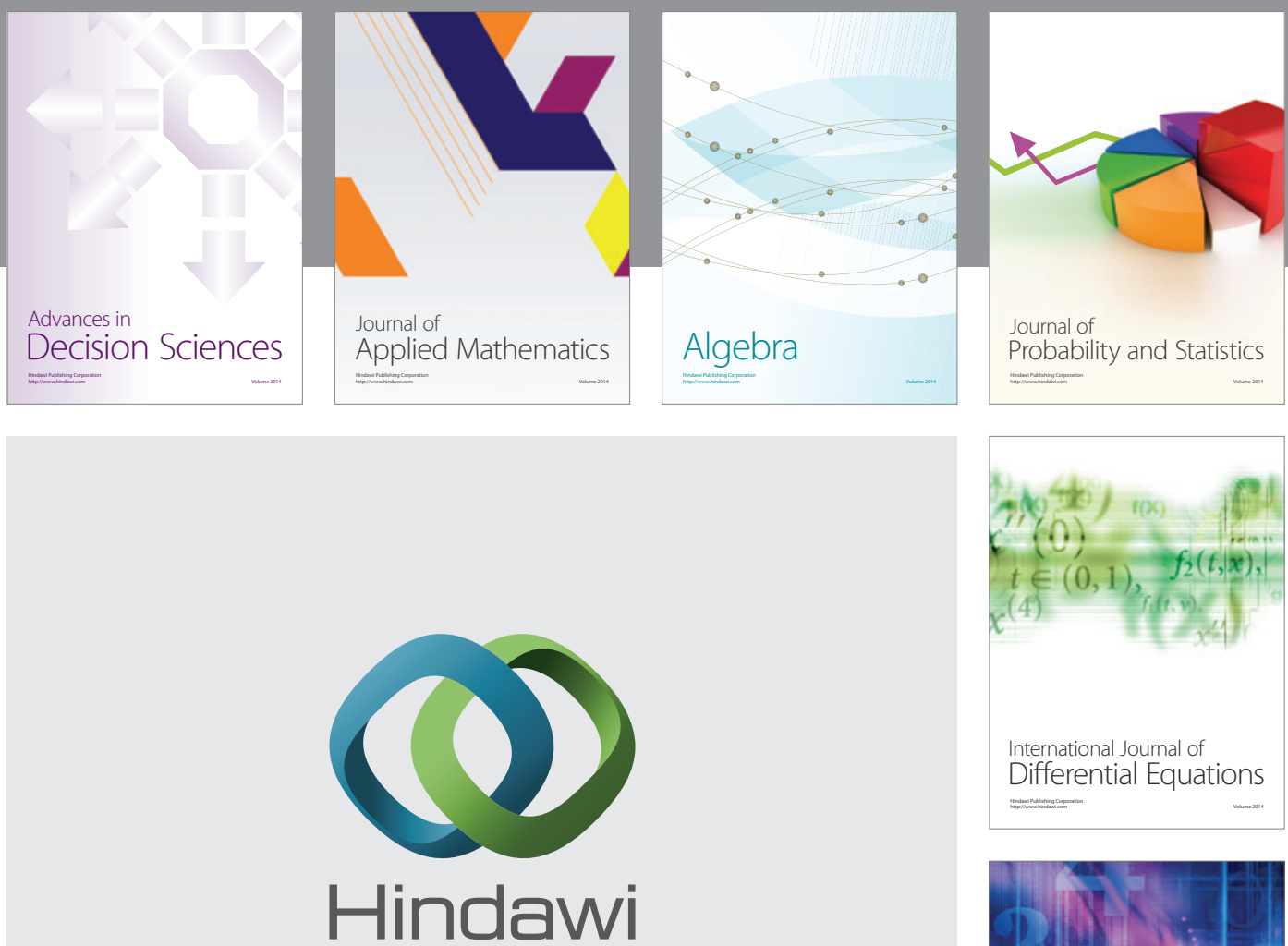

Submit your manuscripts at http://www.hindawi.com
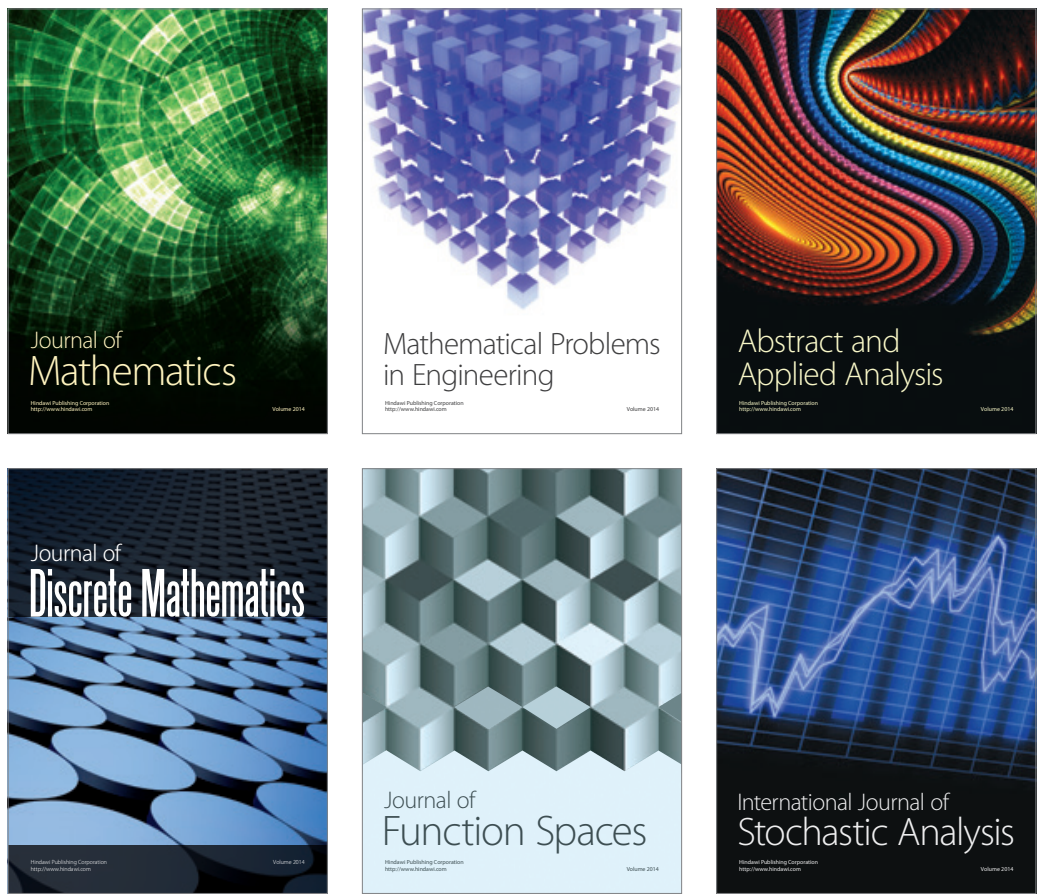

Journal of

Function Spaces

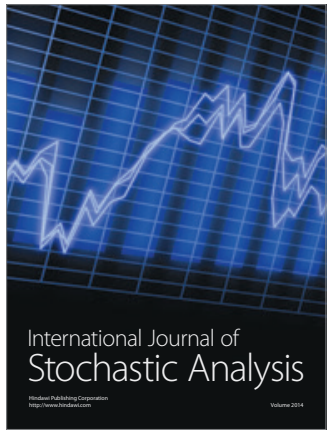

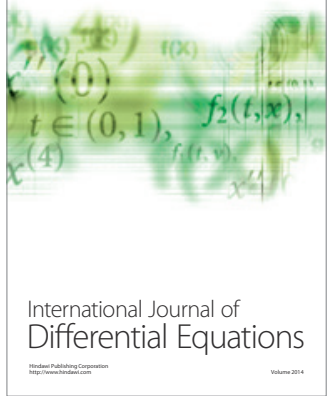
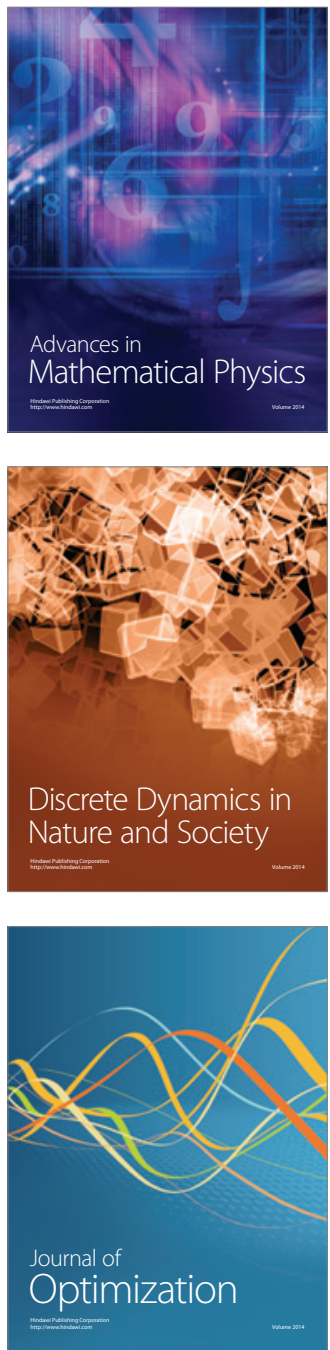